\title{
Comparison theorem of 1-dimensional stochastic hybrid systems
}

\author{
Zhe Yang $^{1}$, Xuerong Mao $^{2}$, Chenggui Yuan ${ }^{3}$ \\ ${ }^{1}$ Institute of Mathematics \\ Shandong University, Jiana 250100, China \\ 2 Department of Statistics and Modelling Science \\ University of Strathclyde, Glasgow G1 1XH, U.K. \\ 3 Department of Mathematics \\ University of Wales Swansea, Swansea SA2 8PP, U.K.
}

\begin{abstract}
In this paper, the comparison theorem of stochastic differential delay equations with Markovian switching has been investigated. In order to develop our theory, a new proof of existence and uniqueness of stochastic differential equations with Markovian switching is given.
\end{abstract}

Keywords: Comparison theorem; Stochastic differential delayed equations with Markovian switching; Brownian motion; Markov chain.

\section{Introduction}

In the past 40 years, the comparison theorems of two Itô's stochastic differential equations have received a lot of attention, for example, Anderson [1], Gal'cuk and Davis [4], Ikeda and Watanable [6], Mao [10], Skorohod [14], Yamada [18] and Yan [19] gave some sufficient conditions for comparison theorem. Recently, Peng and Zhu [7] has presented a sufficient and necessary condition for comparison theorem by using viability theory. However, so far, there is no result for comparison theorem on stochastic differential delay equations, which the present paper shall copy with. Should our theory be more applicable, we shall establish the comparison theorem of stochastic differential delay equations with Markovian switching (SDDEwMSs).

Stochastic differential equations with Markovian switching is an important class of hybrid systems. In ecology, engineering and other disciplines it is well known that many systems exhibit such discrete dynamics, due for example to component failures or repairs, changing subsystem interconnections, etc. When coupled with continuous dynamics, these discrete phenomena give rise to what are known as hybrid systems. Of particular interest to the present paper are hybrid systems whose discrete behavior is driven by continuoustime Markov chains. Such systems have been used to model a number of engineering (and other) systems. For example, Kazangey and Sworder [9] developed a macroeconomics model of the national economy in this framework, to study the effect of federal 
housing removal policies on the stabilization of the housing sector. The term describing the influence of interest rates was modeled by a finite-state Markov chain to provide a quantitative measure of the effect of interest rate uncertainty on the optimal policy. Athans [2] suggested that such hybrid systems would also become a basic framework in posing and solving control-related problems in Battle Management Command, Control and Communications $\left(\mathrm{BM} / \mathrm{C}^{3}\right)$ systems. Hybrid systems were also considered for modeling electric power systems (Willsky and Levy [17]), the control of a solar thermal central receiver (Sworder and Rogers [16]) and the modeling of subtilin production by Bacillus subtilis ( $\mathrm{Hu}, \mathrm{Wu}$ and Sastry [5]). In his book [12], Mariton discussed how such hybrid systems have also emerged as a convenient mathematical framework for the formulation of various design problems in target tracking, fault tolerant control and manufacturing processes. On the other hand, control engineering intuition suggests that time-delays are common in practical systems and are often the cause of instability and/or poor performance. Moreover, it is usually difficult to obtain accurate values for the delay and conservative estimates often have to be used. The importance of time delay has already motivated several studies on the stability of switching diffusions with time delay, see, for example, $[8,11,13]$.

In this paper we compare the following two one-dimensional SDDEwMSs

$$
\left\{\begin{aligned}
d X^{(1)}(t) & =f_{1}\left(X^{(1)}(t), X^{(1)}(t-\delta(t)), t, r(t)\right) d t+g_{1}\left(X^{(1)}(t), X^{(1)}(t-\delta(t)), t, r(t)\right) d B(t), \\
& t \in[0, T], \\
X^{(1)}(t)=x_{1}(t), \quad t & \in[-\tau, 0],
\end{aligned}\right.
$$

and

$$
\left\{\begin{aligned}
d X^{(2)}(t)= & f_{2}\left(X^{(2)}(t), X^{(2)}(t-\delta(t)), t, r(t)\right) d t+g_{2}\left(X^{(2)}(t), X^{(2)}(t-\zeta(t)), t, r(t)\right) d B(t), \\
& t \in[0, T], \\
X^{(2)}(t)=x_{2}(t), \quad t & \in[-\tau, 0],
\end{aligned}\right.
$$

where $r(t), t \geq 0$ is a right-continuous Markov chain on the probability space satisfying certain property. We are interested in the following problem: If $f_{1} \geq f_{2}, g_{1} \geq g_{2}$, we wonder the following is true or not:

$$
x_{1}(s) \geq x_{2}(s), \quad s \in[-\tau, 0], P-\text { a.s.. } \Rightarrow X^{(1)}(t) \geq X^{(1)}(t), \quad t \in[0, T], P-\text { a.s.. }
$$

To treat this problem, we introduce a new type of stochastic differential equations with Markovian switching (SDEwMSs) with stochastic coefficients $f, g$ while the classical $f, g$ are deterministic. In section 2 the existence and uniqueness of the solution to the new equation and the comparison theorem will be given. In section 3 we will get comparison theorem of 1-dimensional classical SDDEwMSs and the approach can also be applied to comparison theorem of 1-dimensional new SDDEwMSs corresponding to the equations in Section 2.

\section{Comparison theorem of SDEwMSs}

Throughout this paper, unless otherwise specified, we let $\left(\Omega, \mathcal{F},\left\{\mathcal{F}_{t}\right\}_{t \geq 0}, P\right)$ be a complete probability space with a filtration $\left\{\mathcal{F}_{t}\right\}_{t \geq 0}$ satisfying the usual conditions (i.e. it is increasing and right continuous while $\mathcal{F}_{0}$ contains all $P$-null sets). Let $B(t)=$ 
$\left(B^{1}(t), \ldots, B^{d}(t)\right)^{T}$ be an $d$-dimensional Brownian motion defined on the probability space. Let $|\cdot|$ denote the Euclidean norm in $\mathbb{R}^{m}$. Let $\tau>0$ and $C\left([-\tau, 0] ; \mathbb{R}^{m}\right)$ denote the family of continuous function $\varphi$ from $[-\tau, 0]$ to $\mathbb{R}^{m}$ with the norm $\|\varphi\|=\sup _{-\tau \leq \theta \leq 0}|\varphi(\theta)|$.

Let $r(t), t \geq 0$, be a right-continuous Markov chain on the probability space taking values in a finite state space $\mathbb{S}=\{1,2, \ldots, N\}$ with generator $\Gamma=\left(\gamma_{i j}\right)_{N \times N}$ given by

$$
P\{r(t+\Delta)=j \mid r(t)=i\}= \begin{cases}\gamma_{i j} \Delta+o(\Delta), & \text { if } i \neq j \\ 1+\gamma_{i j} \Delta+o(\Delta), & \text { if } i=j\end{cases}
$$

where $\Delta>0$. Here $\gamma_{i j}>0$ is transition rate from $i$ to $j$ if $i \neq j$ while

$$
\gamma_{i i}=-\sum_{i \neq j} \gamma_{i j}
$$

We assume that the Markov chain $r(\cdot)$ is independent of the Brownian motion $B(\cdot)$. It is well known that almost every sample path of $r(t)$ is right continuous step function and $r(t)$ is ergodic Markov chain and that there is a sequence $\left\{\tau_{k}\right\}_{k \geq 0}$ of stopping times such that $0=\tau_{0}<\tau_{1}<\ldots \tau_{k} \rightarrow \infty$ and $r(t)$ is a constant on every interval $\left[\tau_{k}, \tau_{k+1}\right)$, i.e., for every $k \geq 0, r(t)=r\left(\tau_{k}\right)$ on $t \in\left[\tau_{k}, \tau_{k+1}\right)$ (see [15]).

Consider a SDEwMSs of the form

$$
d X(t)=f(X(t), t, r(t)) d t+g(X(t), t, r(t)) d B(t)
$$

on $0 \leq t \leq T$ with initial data $X(0)=x_{0} \in L^{2}\left(\mathcal{F}_{0}, \mathbb{R}^{m}\right)$ and $r(0)=i_{0} \in \mathbb{S}$, where $f: \Omega \times \mathbb{R}^{m} \times[0, T] \times \mathbb{S} \rightarrow \mathbb{R}^{m}, g: \Omega \times \mathbb{R}^{m} \times[0, T] \times \mathbb{S} \rightarrow \mathbb{R}^{m \times d}$ satisfy the following conditions

(H1) There exists a constant $L>0$, such that for all $t \in[0, T], i \in \mathbb{S}, x, x^{\prime} \in \mathbb{R}^{m}$,

$$
\left|f(x, t, i)-f\left(x^{\prime}, t, i\right)\right|+\left|g(x, t, i)-g\left(x^{\prime}, t, i\right)\right| \leq L\left|x-x^{\prime}\right|
$$

$$
f(0, \cdot, i) \in L_{\mathcal{F}}^{2}\left(0, T ; \mathbb{R}^{m}\right), g(0, \cdot, i) \in L_{\mathcal{F}}^{2}\left(0, T ; \mathbb{R}^{m \times d}\right), \forall i \in \mathbb{S}
$$

Theorem 2.1 Assume that $f, g$ satisfy $(H 1)$ and $(H 2)$. Then for any initial value $x_{0} \in$ $L^{2}\left(\mathcal{F}_{0}, \mathbb{R}^{m}\right)$ there exists a unique solution $X . \in L_{\mathcal{F}}^{2}\left(0, T ; \mathbb{R}^{m}\right)$ to equation (2.1) and $X$ is $t$-continuous on $[0, T]$.

Proof. We first consider equation (2.1) on $t \in\left[0, \tau_{1} \wedge T\right]$. Fix $\beta=2 L^{2}+2 L \sqrt{L^{2}+2}>0$, where $L$ is the Lipschitz constant given in $(H 1)$. To this $\beta$, we introduce a norm in the Hilbert space $L_{\mathcal{F}}^{2}\left(0, \tau_{1} \wedge T ; \mathbb{R}^{m}\right)$ :

$$
\|\nu(\cdot)\|_{-\beta}=\left(E\left[\int_{0}^{\tau_{1} \wedge T}|\nu(s)|^{2} e^{-\beta s} d s\right]\right)^{\frac{1}{2}} .
$$

Clearly it is equivalent to the original norm of $L_{\mathcal{F}}^{2}\left(0, \tau_{1} \wedge T ; \mathbb{R}^{m}\right)$. But this norm is more convenient to construct a contraction mapping in order to apply the fixed point theorem. We define a mapping

$$
h(x): L_{\mathcal{F}}^{2}\left(0, \tau_{1} \wedge T ; \mathbb{R}^{m}\right) \longrightarrow L_{\mathcal{F}}^{2}\left(0, \tau_{1} \wedge T ; \mathbb{R}^{m}\right),
$$


such that

$$
h(x)(t)=x_{0}+\int_{0}^{t} f\left(x(s), s, i_{0}\right) d s+\int_{0}^{t} g\left(x(s), s, i_{0}\right) d B(s), \quad t \in\left[0, \tau_{1} \wedge T\right] .
$$

Then for any $x(\cdot), x^{\prime}(\cdot) \in L_{\mathcal{F}}^{2}\left(0, \tau_{1} \wedge T ; \mathbb{R}^{m}\right)$, we get

$$
\begin{aligned}
h(x)(t)-h\left(x^{\prime}\right)(t)= & \int_{0}^{t}\left(f\left(x(s), s, i_{0}\right)-f\left(x^{\prime}(s), s, i_{0}\right)\right) d s \\
& +\int_{0}^{t}\left(g\left(x(s), s, i_{0}\right)-g\left(x^{\prime}(s), s, i_{0}\right)\right) d B(s), \quad t \in\left[0, \tau_{1} \wedge T\right] .
\end{aligned}
$$

Applying the generalized Itô's formula (see [15]) to $\left|h(x)(t)-h\left(x^{\prime}\right)(t)\right|^{2} e^{-\beta t}$, and taking expectation, we obtain

$$
\begin{aligned}
& E\left[\left|h(x)(t)-h\left(x^{\prime}\right)(t)\right|^{2} e^{-\beta t}\right] \\
= & E\left[\int_{0}^{t} e^{-\beta s}(-\beta)\left|h(x)(s)-h\left(x^{\prime}\right)(s)\right|^{2} d s\right] \\
+ & E\left[\int_{0}^{t} e^{-\beta s}\left|g\left(x(s), s, i_{0}\right)-g\left(x^{\prime}(s), s, i_{0}\right)\right|^{2} d s\right] \\
+ & 2 E\left[\int_{0}^{t} e^{-\beta s}\left(f\left(x(s), s, i_{0}\right)-f\left(x^{\prime}(s), s, i_{0}\right), h(x)(s)-h\left(x^{\prime}\right)(s)\right) d s\right] .
\end{aligned}
$$

This, together with (H1), yields

$$
\begin{aligned}
& E\left[\int_{0}^{t} \beta e^{-\beta s}\left|h(x)(s)-h\left(x^{\prime}\right)(s)\right|^{2} d s\right] \\
\leq & E\left[\int_{0}^{t} e^{-\beta s}\left|g\left(x(s), s, i_{0}\right)-g\left(x^{\prime}(s), s, i_{0}\right)\right|^{2} d s\right] \\
+ & 2 E\left[\int_{0}^{t} e^{-\beta s}\left(f\left(x(s), s, i_{0}\right)-f\left(x^{\prime}(s), s, i_{0}\right), h(x)(s)-h\left(x^{\prime}\right)(s)\right) d s\right] \\
\leq & E\left[\int_{0}^{t} e^{-\beta s}\left(\left(\frac{2 L^{2}}{\beta}+L^{2}\right)\left|x(s)-x^{\prime}(s)\right|^{2}+\frac{\beta}{2}\left|h(x)(s)-h\left(x^{\prime}\right)(s)\right|^{2}\right) d s\right] .
\end{aligned}
$$

Since $\beta=2 L^{2}+2 L \sqrt{L^{2}+2}$,

$$
E\left[\int_{0}^{\tau_{1} \wedge T} e^{-\beta s}\left|h(x)(s)-h\left(x^{\prime}\right)(s)\right|^{2} d s\right] \leq \frac{1}{2} E\left[\int_{0}^{\tau_{1} \wedge T} e^{-\beta s}\left|x(s)-x^{\prime}(s)\right|^{2} d s\right] .
$$

Thus $h$ is a strict contraction mapping of $L_{\mathcal{F}}^{2}\left(0, \tau_{1} \wedge T ; \mathbb{R}^{m}\right)$. It follows by the fixed point theorem that equation $(2.1)$ has a unique solution $X(\cdot) \in L_{\mathcal{F}}^{2}\left(0, \tau_{1} \wedge T ; \mathbb{R}^{m}\right)$. Moreover, $X$ is $t$-continuous on $\left[0, \tau_{1} \wedge T\right]$ with probability one and $E\left[\int_{0}^{\tau_{1} \wedge T}\left|X_{s}\right|^{2} d s\right]<\infty$.

We now consider equation (2.1) on $t \in\left[\tau_{1} \wedge T, \tau_{2} \wedge T\right]$. Following the same path of the above proof, we can obtain that equation (2.1) has a unique solution $X . \in L_{\mathcal{F}}^{2}\left(\tau_{1} \wedge\right.$ $T, \tau_{2} \wedge T ; \mathbb{R}^{m}$ ) and $X$ is $t$-continuous on $\left[\tau_{1} \wedge T, \tau_{2} \wedge T\right]$ with probability one. Repeating this procedure we get equation (2.1) has a unique continuous solution $X(t)$ on $[0, T]$.

For the future use, we cite the following Lemmas.

Lemma 2.2 Let $X(t)=X(0)+M(t)+V(t)$ be a continuous semimartingale, where $M$ is a continuous local martingale with $M(0)=0$ and $V$ is a continuous process of finite variation with $V(0)=0$, then

$$
X^{+^{2}}(t)=X^{+^{2}}(0)+2 \int_{0}^{t} X^{+}(s) d M(s)+2 \int_{0}^{t} X^{+}(s) d V(s)+\int_{0}^{t} I_{[X(s)>0]} d\langle M, M\rangle(s) .
$$


For the details of Lemma 2.2 see Cao and Yan [3]. The following Lemma is necessary and sufficient condition for comparison theorem of 1-dimensional SDEs [7].

Lemma 2.3 Consider the following two 1-dimensional SDEs defined on $[0, T]$, $j=1,2$

$$
X^{(j)}(t)=x_{j}+\int_{0}^{t} b_{j}\left(s, X^{(j)}(s)\right) d s+\int_{0}^{t} \sigma_{j}\left(s, X^{(j)}(s)\right) d B(s) .
$$

We assume that, for $j=1,2$, that $x_{j} \in \mathbb{R}, b_{j}:[0, T] \times \mathbb{R} \rightarrow \mathbb{R}, \sigma_{j}:[0, T] \times \mathbb{R} \rightarrow \mathbb{R}^{1 \times d}$ are continuous in $(t, x)$ and that there exists a constant $\mu>0$ such that for each $x, x^{\prime} \in \mathbb{R}$ and $t \geq 0$,

$$
\left|b_{j}(t, x)-b_{j}\left(t, x^{\prime}\right)\right| \leq \mu\left|x-x^{\prime}\right|, \quad \quad\left|\sigma_{j}(t, x)-\sigma_{j}\left(t, x^{\prime}\right)\right| \leq \mu\left|x-x^{\prime}\right| .
$$

Then the following conditions are equivalent:

(a) $x_{1} \geq x_{2} \Rightarrow X_{t}^{(1)} \geq X_{t}^{(2)}, \forall t \in[0, T], P$-a.s.;

(b) For each $(t, x) \in[0, T] \times \mathbb{R}, \sigma_{1}(t, x)=\sigma_{2}(t, x), b_{1}(t, x) \geq b_{2}(t, x)$.

In Lemma 2.3, the coefficients of SDEs are deterministic, while in the following lemma, the coefficients of SDEs are random.

Lemma 2.4 Set $m=1$. Consider the following two 1-dimensional SDEs

$$
X^{(1)}(t)=x_{0}+\int_{0}^{t}\left(f\left(X^{(1)}(s), s\right)+b_{s}\right) d s+\int_{0}^{t} g\left(X^{(1)}(s), s\right) d B(s), \quad t \in[0, T]
$$

and

$$
X^{(2)}(t)=x_{0}+\int_{0}^{t} f\left(X^{(2)}(s), s\right) d s+\int_{0}^{t} g\left(X^{(2)}(s), s\right) d B(s), \quad t \in[0, T] .
$$

Assume that $f, g$ satisfy $(H 1),(H 2)$ and $f, g$ are continuous in $t, b \in L_{\mathcal{F}}^{2}(0, T ; \mathbb{R})$. If $b \geq 0$, then $\forall x_{0} \in L^{2}\left(\mathcal{F}_{0}, \mathbb{R}\right)$, we have $X^{(1)}(t) \geq X^{(2)}(t), \forall t \in[0, T], P-a . s$.

Proof. By Lemma 2.2, we get

$$
\begin{aligned}
& E\left[\left(X^{(2)}(t)-X^{(1)}(t)\right)^{+^{2}}\right] \\
= & 2 E\left[\int_{0}^{t}\left(X^{(2)}(s)-X^{(1)}(s)\right)^{+}\left(f\left(X^{(2)}(s), s\right)-f\left(X^{(1)}(s), s\right)-b_{s}\right) d s\right] \\
+ & E\left[\int_{0}^{t} \mathbb{I}_{\left[X^{(2)}(s)-X^{(1)}(s)>0\right]}\left(g\left(X^{(2)}(s), s\right)-g\left(X^{(1)}(s), s\right)\right)^{2} d s\right] \\
\leq & 2 E\left[\int_{0}^{t}\left(X^{(2)}(s)-X^{(1)}(s)\right)^{+}\left(f\left(X^{(2)}(s), s\right)-f\left(X^{(1)}(s), s\right)\right) d s\right] \\
+ & E\left[\int_{0}^{t} \mathbb{I}_{\left[X^{(2)}(s)-X^{(1)}(s)>0\right]}\left(g\left(X^{(2)}(s), s\right)-g\left(X^{(1)}(s), s\right)\right)^{2} d s\right] \\
\leq & E\left[\left(2 L+L^{2}\right) \int_{0}^{t}\left(X^{(2)}(s)-X^{(1)}(s)\right)^{+^{2}} d s\right]
\end{aligned}
$$

which implies that $E\left[\left(X^{(2)}(t)-X^{(1)}(t)\right)^{+^{2}}\right]=0, \forall t \in[0, T]$, by Grownwall's inequality, that is, $X^{(1)}(t) \geq X^{(2)}(t), \forall t \in[0, T], \mathrm{P}-$ a.s..

In the following, we shall investigate the comparison theorem of the SDEwMSs. 
Theorem 2.5 Set $m=1$. Consider the following two 1-dimensional SDEwMSs

$$
X^{(1)}(t)=x_{0}^{(1)}+\int_{0}^{t} f_{1}\left(X^{(1)}(s), s, r(s)\right) d s+\int_{0}^{t} g\left(X^{(1)}(s), s, r(s)\right) d B(s), \quad t \in[0, T]
$$

and

$$
X^{(2)}(t)=x_{0}^{(2)}+\int_{0}^{t} f_{2}\left(X^{(2)}(s), s, r(s)\right) d s+\int_{0}^{t} g\left(X^{(2)}(s), s, r(s)\right) d B(s), \quad t \in[0, T] .
$$

Assume that $f_{j}, g$ satisfy $(H 1),(H 2)$ and $f_{j}, g$ are continuous in $t, j=1,2$. If for a.a. $\omega$, $f_{1}(x, t, i) \geq f_{2}(x, t, i), \forall(x, t, i) \in[0, T] \times \mathbb{R} \times \mathbb{S}, x_{0}^{(1)} \geq x_{0}^{(2)}$, we have $X^{(1)}(t) \geq X^{(2)}(t), \forall t \in$ $[0, T], P-a . s$.

Proof. We consider the above two equations on $t \in\left[0, \tau_{1} \wedge T\right]$. Denote the differences by $\hat{X}(\cdot)=X^{(1)}(\cdot)-X^{(2)}(\cdot), x_{0}=x_{0}^{(1)}-x_{0}^{(2)}$. Then

$$
\begin{aligned}
& \hat{X}(t) \\
= & x_{0}+\int_{0}^{t}\left(f_{1}\left(X^{(1)}(s), s, i_{0}\right)-f_{2}\left(X^{(2)}(s), s, i_{0}\right)\right) d s \\
+ & \int_{0}^{t}\left(g\left(X^{(1)}(s), s, i_{0}\right)-g\left(X^{(2)}(s), s, i_{0}\right)\right) d B(s) \\
= & x_{0}+\int_{0}^{t}\left[f_{s} \hat{X}(s)+\left(f_{1}\left(X^{(1)}(s), s, i_{0}\right)-f_{2}\left(X^{(1)}(s), s, i_{0}\right)\right)\right] d s+\int_{0}^{t} g_{s} \hat{X}(s) d B(s),
\end{aligned}
$$

where

$$
\begin{aligned}
& f_{s}= \begin{cases}\frac{f_{2}\left(X^{(1)}(s), s, i_{0}\right)-f_{2}\left(X^{(2)}(s), s, i_{0}\right)}{X^{(1)}(s)-X^{(2)}(s)}, & \text { if } X^{(1)}(s) \neq X^{(2)}(s) ; \\
0, & \text { if } X^{(1)}(s)=X^{(2)}(s),\end{cases} \\
& g_{s}= \begin{cases}\frac{g\left(X^{(1)}(s), s, i_{0}\right)-g\left(X^{(2)}(s), s, i_{0}\right)}{X^{(1)}(s)-X^{(2)}(s)}, & \text { if } X^{(1)}(s) \neq X^{(2)}(s) ; \\
0, & \text { if } X^{(1)}(s)=X^{(2)}(s) .\end{cases}
\end{aligned}
$$

Since $f_{1}\left(X^{(1)}(t), t, i_{0}\right) \geq f_{2}\left(X^{(1)}(t), t, i_{0}\right), t \in\left[0, \tau_{1} \wedge T\right]$, by Lemma 2.4 we know $\hat{X}(t) \geq$ $\bar{X}(t), t \in\left[0, \tau_{1} \wedge T\right]$, a.e., where $\bar{X}(\cdot)$ is the solution of the SDE

$$
\bar{X}(t)=x_{0}+\int_{0}^{t} f_{s} \bar{X}(s) d s+\int_{0}^{t} g_{s} \bar{X}(s) d B(s) .
$$

Note $\bar{X}(t)=x_{0} \exp \left[\int_{0}^{t}\left(f_{s}-\frac{1}{2}\left|g_{s}\right|^{2}\right) d s+\int_{0}^{t} g_{s} d B(s)\right] \geq 0, t \in\left[0, \tau_{1} \wedge T\right]$, we obtain $X^{(1)}(t) \geq$ $X^{(2)}(t), \forall t \in[0, T], \mathrm{P}$-a.s.. Repeat the same procedure above, we shall get $X^{(1)}(t) \geq$ $X^{(2)}(t), t \in\left[\tau_{i} \wedge T, \tau_{i+1} \wedge T\right], i \geq 1$ a.s. Therefore the proof is complete.

\section{Comparison theorem of 1-dimensional SDDEwMSs}

In this section $\delta(\cdot)$ and $\zeta(\cdot)$ are two nonnegative continuous real-valued functions defined on $[0, T]$ satisfying

(A1) there exists a constant $\tau>0$ such that for each $t \in[0, T]$,

$$
-\tau \leq t-\delta(t) \leq t, \quad-\tau \leq t-\zeta(t) \leq t
$$


(A2) there exists a constant $L>0$ such that for each $t \in[0, T]$, and for each nonnegative and integrable $\xi(\cdot)$,

$$
\int_{0}^{t} \xi(s-\delta(s)) d s \leq L \int_{-\tau}^{t} \xi(s) d s ; \quad \int_{0}^{t} \xi(s-\zeta(s)) d s \leq L \int_{-\tau}^{t} \xi(s) d s .
$$

Remark 3.1 Examples for $\delta(s)$ satisfying $(A 1)$ and $(A 2)$. Let $\delta(s) \equiv c$, where $c>0$ is a constant. Or let $s-\delta(s)$ be a monotone nonnegative function whose converse function has continuous differential function.

We now consider the comparison theorem of 1-dimensional classical SDDEwMSs. Look at the following two equations

$$
\left\{\begin{aligned}
d X^{(1)}(t) & =f_{1}\left(X^{(1)}(t), X^{(1)}(t-\delta(t)), t, r(t)\right) d t+g_{1}\left(X^{(1)}(t), X^{(1)}(t-\zeta(t)), t, r(t)\right) d B(t), \\
& t \in[0, T], \\
X^{(1)}(t) & =x_{1}(t), \quad t \in[-\tau, 0],
\end{aligned}\right.
$$

and

$$
\left\{\begin{aligned}
d X^{(2)}(t) & =f_{2}\left(X^{(2)}(t), X^{(2)}(t-\delta(t)), t, r(t)\right) d t+g_{2}\left(X^{(2)}(t), X^{(2)}(t-\zeta(t)), t, r(t)\right) d B(t), \\
& t \in[0, T], \\
X^{(2)}(t) & =x_{2}(t), \quad t \in[-\tau, 0],
\end{aligned}\right.
$$

where for $j=1,2,\left\{x_{j}(t):-\tau \leq t \leq 0\right\} \in C([-\tau, 0] ; \mathbb{R})$ are both $\mathcal{F}_{0}$-measurable, $r(\cdot)$ is the same as in Section $2, f_{j}: \mathbb{R} \times \mathbb{R} \times[0, T] \times \mathbb{S} \rightarrow \mathbb{R}$ and $g_{j}: \mathbb{R} \times \mathbb{R} \times[0, T] \times \mathbb{S} \rightarrow \mathbb{R}^{1 \times d}$ satisfy the following conditions

(H3) There exists a constant $K>0$, such that for all $t \in[0, T], i \in \mathbb{S}, x, y, x^{\prime}, y^{\prime} \in \mathbb{R}$,

$$
\begin{gathered}
\left|f_{1}(x, y, t, i)-f_{1}\left(x^{\prime}, y^{\prime}, t, i\right)\right| \vee\left|f_{2}(x, y, t, i)-f_{2}\left(x^{\prime}, y^{\prime}, t, i\right)\right| \\
\vee\left|g_{1}(x, y, t, i)-g_{1}\left(x^{\prime}, y^{\prime}, t, i\right)\right| \vee\left|g_{2}(x, y, t, i)-g_{2}\left(x^{\prime}, y^{\prime}, t, i\right)\right| \leq K\left(\left|x-x^{\prime}\right|+\left|y-y^{\prime}\right|\right) ;
\end{gathered}
$$

and there is, moreover, a $h>0$ such that for all $t \in[0, T], x, y, x^{\prime}, y^{\prime} \in \mathbb{R}$,

$$
\left|f_{1}(x, y, t, i)\right|+\left|f_{2}(x, y, t, i)\right|+\left|g_{1}(x, y, t, i)\right|+\left|g_{2}(x, y, t, i)\right| \leq h(1+|x|+|y|) .
$$

Under hypothesis $(H 3),(A 1)$ and $(A 2)$, using the similar method to the proof of Theorem 2.1, we can get each of the above two SDDEwMSs has a unique continuous solution. Firstly, we shall give two simple examples which both counter the comparison theorem. One is for the case where $f_{2}$ is nonincreasing in $y$ and the other is for $g$ has $y$ term.

Example 3.2 Given $T>0, \tau>0$, we consider the following two SDDEs

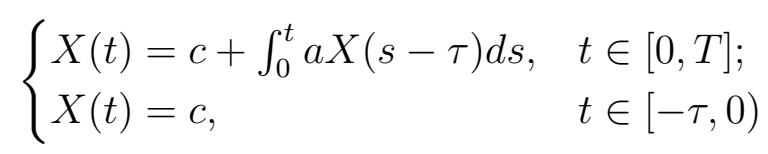

and

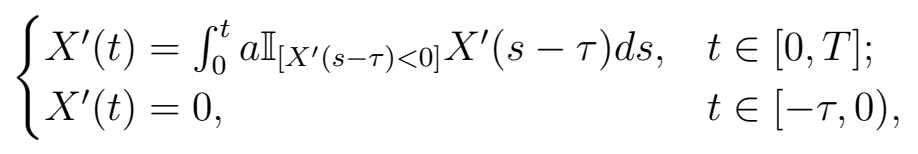

where $a=-\frac{2}{\tau}, c<0$ are given constants. It is obvious that ay $\leq a y \mathbb{I}_{[y<0]}, X^{\prime} \equiv 0$. But $X(t)>0, t \in\left(\frac{2}{\tau}, \tau\right]$. 
Example 3.3 Given $T>0, \tau>0$, we consider the following two SDDEs

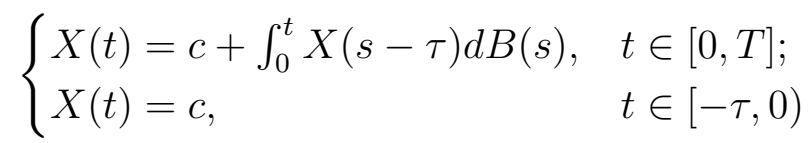

and

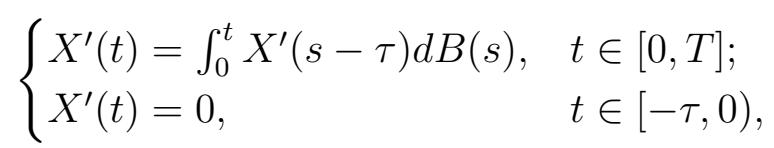

where $c<0$ is a given constant. We can easily get $X^{\prime} \equiv 0$. When $t \in[0, \tau]$, we have $X(t)=c+c B(t)$. But

$$
P\{(\omega, t) \in \Omega \times[0, \tau]: X(\omega, t)>0\}>0 .
$$

From the example above, we know that the comparison theorem does not hold for which the diffusion contains delay. In the following, we shall give a sufficient condition of the comparison theorem for a class of SDDEwMS.

Theorem 3.4 Consider the following two 1-dimensional SDDEwMSs

$$
\left\{\begin{aligned}
d X^{(1)}(t) & =f_{1}\left(X^{(1)}(t), X^{(1)}(t-\delta(t)), t, r(t)\right) d t+g\left(X^{(1)}(t), t, r(t)\right) d B(t), t \in[0, T] \\
X^{(1)}(t) & =x_{1}(t), \quad t \in[-\tau, 0]
\end{aligned}\right.
$$

and

$$
\left\{\begin{aligned}
d X^{(2)}(t) & =f_{2}\left(X^{(2)}(t), X^{(2)}(t-\delta(t)), t, r(t)\right) d t+g\left(X^{(2)}(t), t, r(t)\right) d B(t), t \in[0, T] \\
X^{(2)}(t) & =x_{2}(t), \quad t \in[-\tau, 0] .
\end{aligned}\right.
$$

Assume $\delta(t)$ satisfy $(A 1),(A 2)$ and

(H1') There exists a constant $C>0$, such that for all $t \in[0, T], i \in \mathbb{S}, x, y, x^{\prime}, y^{\prime} \in \mathbb{R}$,

$$
\begin{gathered}
\left|f_{1}(x, y, t, i)-f_{1}\left(x^{\prime}, y^{\prime}, t, i\right)\right| \vee\left|f_{2}(x, y, t, i)-f_{2}\left(x^{\prime}, y^{\prime}, t, i\right)\right| \leq C\left(\left|x-x^{\prime}\right|+\left|y-y^{\prime}\right|\right), \\
\left|g(x, t, i)-g\left(x^{\prime}, t, i\right)\right| \leq C\left|x-x^{\prime}\right|
\end{gathered}
$$

and there is, moreover, a $\bar{h}>0$ such that for all $t \in[0, T], x, y, x^{\prime}, y^{\prime} \in \mathbb{R}$,

$$
\left|f_{1}(x, y, t, i)\right|+\left|f_{2}(x, y, t, i)\right| \leq \bar{h}(1+|x|+|y|), \quad|g(x, t, i)| \leq \bar{h}(1+|x|) ;
$$

(H2') $f_{1}, f_{2}$ are continuous in $(x, y, t), g$ is continuous in $(x, t), f_{2}$ is increasing in $y$, i.e. $f_{2}(x, y, t, i) \geq f_{2}\left(x, y^{\prime}, t, i\right), \forall x \in \mathbb{R}, t \in[0, T], i \in \mathbb{S}$, if $y \geq y^{\prime}$.

If $f_{1}(x, y, t, i) \geq f_{2}(x, y, t, i), t \in[0, T], x, y \in \mathbb{R}, i \in \mathbb{S}, x_{1}(s) \geq x_{2}(s), s \in[-\tau, 0]$, we have

$$
X^{(1)}(t) \geq X^{(2)}(t), \text { a.e., a.s.. }
$$

Proof. Let $X^{(1)}(\cdot)$ be the solution of equation $(3.1)$ and $X^{(2)}(\cdot)$ be the solution of equation (3.2). We consider the case when $t \in\left[0, \tau_{1} \wedge T\right]$. Set

$$
\left\{\begin{aligned}
d X^{(3)}(t) & =f_{2}\left(X^{(3)}(t), X^{(1)}(t-\delta(t)), t, i_{0}\right) d t+g\left(X^{(3)}(t), t, i_{0}\right) d B(t), & & t \in\left[0, \tau_{1} \wedge T\right] \\
X^{(3)}(t) & =x_{2}(t), & & t \in[-\tau, 0] .
\end{aligned}\right.
$$


It is obvious that there exists a unique solution of the above SDEwMSs by Theorem 2.1. By Theorem 2.5, that $f_{1}\left(x, X^{(1)}(t-\delta(t)), t, i_{0}\right) \geq f_{2}\left(x, X^{(1)}(t-\delta(t)), t, i_{0}\right), t \in$ $\left[0, \tau_{1} \wedge T\right], x \in \mathbb{R}$ and that $x_{1}(0) \geq x_{2}(0)$ imply $X^{(1)}(t) \geq X^{(3)}(t), \forall t \in\left[0, \tau_{1} \wedge T\right], \mathrm{P}-$ a.s. Set

$$
\left\{\begin{aligned}
d X^{(4)}(t) & =f_{2}\left(X^{(4)}(t), X^{(3)}(t-\delta(t)), t, i_{0}\right) d t+g\left(X^{(4)}(t), t, i_{0}\right) d B(t), & & t \in\left[0, \tau_{1} \wedge T\right] ; \\
X^{(4)}(t) & =x_{2}(t), & & t \in[-\tau, 0] .
\end{aligned}\right.
$$

Also by Theorem 2.5, since $f_{2}$ is increasing about $y$ and $f_{2}\left(x, X^{(1)}(t-\delta(t)), t, i_{0}\right) \geq$ $f_{2}\left(x, X^{(3)}(t-\delta(t)), t, i_{0}\right), t \in\left[0, \tau_{1} \wedge T\right], x \in \mathbb{R}$, we have $X^{(3)}(t) \geq X^{(4)}(t), \forall t \in\left[0, \tau_{1} \wedge T\right]$, $\mathrm{P}-$ a.s.

Similarly for $n=5,6, \cdots$, we consider the following SDEwMS

$$
\left\{\begin{aligned}
d X^{(n)}(t) & =f_{2}\left(X^{(n)}(t), X^{(n-1)}(t-\delta(t)), t, i_{0}\right) d t+g\left(X^{(n)}(t), t, i_{0}\right) d B(t), & & t \in\left[0, \tau_{1} \wedge T\right] ; \\
X^{(n)}(t) & =x_{2}(t), & & t \in[-\tau, 0] .
\end{aligned}\right.
$$

Similarly we have $X^{(4)}(t) \geq X^{(5)}(t) \geq \cdots \geq X^{(n)}(t) \geq \cdots, \forall t \in\left[0, \tau_{1} \wedge T\right]$, P-a.s..

We fix $\beta=1+(1+2 L) C^{2}+2 C>0$, where $C$ is the Lipschitz constant given in $\left(H 1^{\prime}\right)$. To this $\beta$, we introduce a norm in the Hilbert space $L_{\mathcal{F}}^{2}\left(0, \tau_{1} \wedge T ; \mathbb{R}\right)$ like in the proof of Theorem 2.1:

$$
\|\nu(\cdot)\|_{-\beta}=\left(E\left[\int_{0}^{\tau_{1} \wedge T}|\nu(s)|^{2} e^{-\beta s} d s\right]\right)^{\frac{1}{2}}
$$

Set $\hat{X}^{(n)}(t)=X^{(n)}(t)-X^{(n-1)}(t), \quad n \geq 4$. Then $\hat{X}^{(n)}(t)$ satisfies

$$
\left\{\begin{array}{rlrl}
d \hat{X}^{(n)}(t)= & \left(f_{2}\left(X^{(n)}(t), X^{(n-1)}(t-\delta(t)), t, i_{0}\right)-f_{2}\left(X^{(n-1)}(t),\right.\right. & \left.\left.X^{(n-2)}(t-\delta(t)), t, i_{0}\right)\right) d t \\
& +\left(g\left(X^{(n)}(t), t, i_{0}\right)-g\left(X^{(n-1)}(t), t, i_{0}\right)\right) d B(t), & & t \in\left[0, \tau_{1} \wedge T\right] ; \\
\hat{X}^{(n)}(t)= & 0, & & t \in[-\tau, 0] .
\end{array}\right.
$$

Applying Itô's formula to $\left|\hat{X}^{(n)}(t)\right|^{2} e^{-\beta t}$, then take expectation, we obtain

$$
\begin{aligned}
& E\left[\left|\hat{X}^{(n)}(t)\right|^{2} e^{-\beta t}\right] \\
= & E\left[\int_{0}^{t} e^{-\beta s}(-\beta)\left|\hat{X}^{(n)}(s)\right|^{2} d s\right]+E\left[\int_{0}^{t} e^{-\beta s}\left(g\left(X^{(n)}(s), s, i_{0}\right)-g\left(X^{(n-1)}(s), s, i_{0}\right)\right)^{2} d s\right] \\
+ & 2 E\left[\int_{0}^{t}\left(f_{2}\left(X^{(n)}(s), X^{(n-1)}(s-\delta(s)), s, i_{0}\right)-f_{2}\left(X^{(n-1)}(s), X^{(n-2)}(s-\delta(s)), s, i_{0}\right)\right)\right. \\
& \left.\cdot e^{-\beta s} \hat{X}^{(n)}(s) d s\right] .
\end{aligned}
$$

Since $f_{2}, g$ satisfy $\left(H 1^{\prime}\right)$,

$$
\begin{aligned}
& E\left[\left|\hat{X}^{(n)}(t)\right|^{2} e^{-\beta t}\right] \\
\leq & E\left[\int_{0}^{t} e^{-\beta s}(-\beta)\left|\hat{X}^{(n)}(s)\right|^{2} d s\right]+C^{2} E\left[\int_{0}^{t} e^{-\beta s}\left|\hat{X}^{(n)}(s)\right|^{2} d s\right] \\
+ & C E\left[\int_{0}^{t} e^{-\beta s} 2 \hat{X}^{(n)}(s)\left(\left|\hat{X}^{(n)}(s)\right|+\left|\hat{X}^{(n-1)}(s-\delta(s))\right|\right) d s\right] \\
\leq & E\left[\int_{0}^{t} e^{-\beta s}\left(C^{2}-\beta+2 C\right)\left|\hat{X}^{(n)}(s)\right|^{2} d s\right] \\
+ & E\left[\int_{0}^{t} e^{-\beta s} 2 C \hat{X}^{(n)}(s)\left|\hat{X}^{(n-1)}(s-\delta(s))\right| d s\right]
\end{aligned}
$$




$$
\begin{aligned}
\leq & E\left[\int_{0}^{t} e^{-\beta s}\left((1+2 L) C^{2}+2 C-\beta\right)\left|\hat{X}^{(n)}(s)\right|^{2} d s\right] \\
& +E\left[\int_{0}^{t} e^{-\beta s} \frac{1}{2 L}\left|\hat{X}^{(n-1)}(s-\delta(s))\right|^{2} d s\right] .
\end{aligned}
$$

Since $\beta=1+(1+2 L) C^{2}+2 C, \delta$ satisfies $(A 2)$, we get

$$
\begin{aligned}
& E\left[\int_{0}^{t} e^{-\beta s}\left|\hat{X}^{(n)}(s)\right|^{2} d s\right] \leq \frac{1}{2 L} E\left[\int_{0}^{t} e^{-\beta s}\left|\hat{X}^{(n-1)}(s-\delta(s))\right|^{2} d s\right] \\
\leq & \frac{1}{2 L} E\left[\int_{0}^{t} e^{-\beta(s-\delta(s))}\left|\hat{X}^{(n-1)}(s-\delta(s))\right|^{2} d s\right] \leq \frac{1}{2} E\left[\int_{-\tau}^{t} e^{-\beta s}\left|\hat{X}^{(n-1)}(s)\right|^{2} d s\right] \\
= & \frac{1}{2} E\left[\int_{0}^{t} e^{-\beta s}\left|\hat{X}^{(n-1)}(s)\right|^{2} d s\right] .
\end{aligned}
$$

Thus

$$
E\left[\int_{0}^{t} e^{-\beta s}\left|\hat{X}^{(n)}(s)\right|^{2} d s\right] \leq\left(\frac{1}{2}\right)^{n-4} E\left[\int_{0}^{t} e^{-\beta s}\left|\hat{X}^{(4)}(s)\right|^{2} d s\right] .
$$

Set $t=\tau_{1} \wedge T$, it follows that $\left(X^{(n)}\right)_{n \geq 4}$ is a Cauchy sequence in $L_{\mathcal{F}}^{2}\left(0, \tau_{1} \wedge T ; \mathbb{R}\right)$. Since $L_{\mathcal{F}}^{2}\left(0, \tau_{1} \wedge T ; \mathbb{R}\right)$ is a Banach space, we obtain $X . \in L_{\mathcal{F}}^{2}\left(0, \tau_{1} \wedge T ; \mathbb{R}\right)$ satisfies the following equation

$$
\left\{\begin{aligned}
d X(t) & =f_{2}\left(X(t), X(t-\delta(t)), t, i_{0}\right) d t+g\left(X(t), t, i_{0}\right) d B(t), & & t \in\left[0, \tau_{1} \wedge T\right] \\
X(t) & =x_{2}(t), & & t \in[-\tau, 0] .
\end{aligned}\right.
$$

By the existence and uniqueness theorem of classical SDDEwMSs, we know

$$
X(t)=X^{(2)}(t), \quad \forall t \in\left[0, \tau_{1} \wedge T\right], \quad P-a . s .
$$

Since $X^{(1)}(t) \geq X^{(3)}(t) \geq X^{(4)}(t) \geq X(t), t \in\left[0, \tau_{1} \wedge T\right]$, it holds immediately

$$
X^{(1)}(t) \geq X^{(2)}(t), \quad \forall t \in\left[0, \tau_{1} \wedge T\right], \quad P-a . s . .
$$

Corollary 3.5 Let $X^{(1)}(\cdot)$ be the solution of equation (3.1) and $X^{(2)}(\cdot)$ be the solution of equation (3.2). Assume $f_{1}, f_{2}, g$ satisfy $\left(H 1^{\prime}\right)$ and $\left(H 2^{\prime}\right), \delta$ satisfy $(A 1)$ and $(A 2)$. If $f_{1}\left(X^{(1)}(t), X^{(1)}(t-\delta(t)), t, i\right) \geq f_{2}\left(X^{(1)}(t), X^{(1)}(t-\delta(t)), t, i\right), t \in[0, T], i \in \mathbb{S}, x_{1}(s) \geq$ $x_{2}(s), s \in[-\tau, 0]$, we have $X^{(1)}(t) \geq X^{(2)}(t), \forall t \in[0, T], P-a . s .$.

Proof. We consider the case when $t \in\left[0, \tau_{1} \wedge T\right]$. Set

$$
\left\{\begin{aligned}
d X^{(3)}(t) & =f_{2}\left(X^{(3)}(t), X^{(1)}(t-\delta(t)), t, i_{0}\right) d t+g\left(X^{(3)}(t), t, i_{0}\right) d B(t), & & t \in\left[0, \tau_{1} \wedge T\right] \\
X^{(3)}(t) & =x_{2}(t), & & t \in[-\tau, 0] .
\end{aligned}\right.
$$

Then

$$
\begin{aligned}
& X^{(1)}(t)-X^{(3)}(t) \\
= & x_{1}(0)-x_{2}(0)+\int_{0}^{t}\left(g\left(X^{(1)}(s), s, i_{0}\right)-g\left(X^{(3)}(s), s, i_{0}\right)\right) d B(s) \\
+ & \int_{0}^{t}\left[f_{1}\left(X^{(1)}(s), X^{(1)}(s-\delta(s)), s, i_{0}\right)-f_{2}\left(X^{(3)}(s), X^{(1)}(s-\delta(s)), s, i_{0}\right)\right] d s \\
= & x_{1}(0)-x_{2}(0)+\int_{0}^{t} g_{s} \cdot\left(X^{(1)}(s)-X^{(3)}(s)\right) d B(s)+\int_{0}^{t} f_{s} \cdot\left(X^{(1)}(s)-X^{(3)}(s)\right) d s \\
+ & \int_{0}^{t}\left[f_{1}\left(X^{(1)}(s), X^{(1)}(s-\delta(s)), s, i_{0}\right)-f_{2}\left(X^{(1)}(s), X^{(1)}(s-\delta(s)), s, i_{0}\right)\right] d s,
\end{aligned}
$$


where

$$
f_{s}=\frac{f_{2}\left(X^{(1)}(s), X^{(1)}(s-\delta(s)), s, i_{0}\right)-f_{2}\left(X^{(3)}(s), X^{(1)}(s-\delta(s)), s, i_{0}\right)}{X^{(1)}(s)-X^{(3)}(s)} \mathbb{I}_{\left\{X^{(1)}(s) \neq X^{(3)}(s)\right\}}
$$

and

$$
g_{s}=\frac{\left.g\left(X^{(1)}(s), s, i_{0}\right)-g\left(X^{(3)}(s), s, i_{0}\right)\right)}{X^{(1)}(s)-X^{(3)}(s)} \mathbb{I}_{\left\{X^{(1)}(s) \neq X^{(3)}(s)\right\}} .
$$

Since $f_{1}\left(X^{(1)}(t), X^{(1)}(t-\delta(t)), t, i_{0}\right) \geq f_{2}\left(X^{(1)}(t), X^{(1)}(t-\delta(t)), t, i_{0}\right), t \in\left[0, \tau_{1} \wedge T\right]$, by Lemma 2.4 we know $X^{(1)}(t)-X^{(3)}(t) \geq \bar{X}(t), t \in\left[0, \tau_{1} \wedge T\right]$, a.e., where $\bar{X}(\cdot)$ is the solution of the SDE

$$
\bar{X}(t)=x_{1}(0)-x_{2}(0)+\int_{0}^{t} f_{s} \bar{X}(s) d s+\int_{0}^{t} g_{s} \bar{X}(s) d B(s) .
$$

Note $\bar{X}(t)=\left(x_{1}(0)-x_{2}(0)\right) \exp \left[\int_{0}^{t}\left(f_{s}-\frac{1}{2}\left|g_{s}\right|^{2}\right) d s+\int_{0}^{t} g_{s} d B(s)\right] \geq 0, t \in\left[0, \tau_{1} \wedge T\right]$, we obtain $X^{(1)}(t) \geq X^{(3)}(t), \forall t \in\left[0, \tau_{1} \wedge T\right]$, P-a.s..

Set

$$
\left\{\begin{aligned}
d X^{(4)}(t) & =f_{2}\left(X^{(4)}(t), X^{(3)}(t-\delta(t)), t, i_{0}\right) d t+g\left(X^{(4)}(t), t, i_{0}\right) d B(t), & & t \in\left[0, \tau_{1} \wedge T\right] \\
X^{(4)}(t) & =x_{2}(t), & & t \in[-\tau, 0] .
\end{aligned}\right.
$$

Similarly to the proof of Theorem 3.4, we get $X^{(1)}(t) \geq X^{(2)}(t), \forall t \in[0, T]$, P-a.s..

\section{Acknowledgements}

The authors wish to thank Professor Shige, Peng for his enthusiastic help.

\section{References}

[1] W.J. Anderson, Local behaviour of solutions of stochastic integral equations, Trans. Amer. Math. Soc. 164 (1972) 309-321.

[2] Athans, M., Command and control (c2) theory: A challenge to control science, IEEE Trans. Automat. Control 32(1987), 286-293.

[3] Z.Cao, J.Yan, A comparison theorem for solutions of stochastic differential equations. Adv. Math. (Chinese) 28, 304-308, 1999.

[4] L.I. Gal'cluk, M.H.A. Davis, A note on a comparison theorem for equations with different diffusions, Stochastics 6 (1982) 147-149.

[5] Hu, J., Wu W. and Sastry S., Modeling subtilin production in bacillus subtilis using stochastic hybrid systems. In R. Alur, G. Pappas Eds., Hybrid Systems: Computation and Control, 7th International Workshop, HSCC 2004, 417-431. Springer LNCS vol. 2993, 2004.

[6] N. Ikeda, S. Watanabe, A comparison theorem for solutions of stochasitic differential equations and its applications, Osaka J. Math. 14 (1977) 619-633. 
[7] S.Peng and X.Zhu, Necessary and sufficient condition for comparison theorem of 1-dimensional stochastic differential equations, Stochastic Processes and their Applications, 116, No.3, 370-380, 2006.

[8] Y. Y. Cao, Y. X. Sun and J. Lam, delay-dependent robust $H_{\infty}$ control for uncertain systems with time-varying delays. IEE Proceeding- Control Theory and Applications, 145(1998), 338-344.

[9] T. Kazangey and D.D. Sworder, Effective federal policies for regulating residential housing, in: Proc. Summer Computer Simulation Conference, San diego, 1971, 11201128.

[10] X. Mao, A note on comparison theorems for stochastic differential equations with respect to semimartingales, Stochastics Stochastics Rep. 37 (1991) 49-59.

[11] X. Mao, A. Matasov and A. B. Piunovskiy, Stochastic differential delay equations with Markovian switching, Bernoulli 6 (2000), 73-90.

[12] Mariton, M., Jump Linear Systems in Automatic Control, Marcel Dekker, New York, 1990.

[13] P. Park, A delay-dependent stability criterion for systems with uncertain linear systems. IEEE Trans. Automat. Control 44(1999).

[14] A.V. Skorokhod, Studies in the Theory of Random Process, Addison-Wesley, Reading, MA, 1965.

[15] A.V. Skorohod, Asymptotic Methods in the Theory of Stochastic Differential Equations, American Mathematical Society, Providence, 1989.

[16] Sworder, D. D. and Rogers, R. O., An LQ-solution to a control problem associated with a solar thermal central receiver, IEEE Trans. Automat. Control 28(1983), 971978.

[17] Willsky, A.S. and Levy, B.C., Stochastic stability research for complex power systems, DOE Contract, LIDS, MIT, Rep. ET-76-C-01-2295, 1979.

[18] T. Yamada, On comparison theorem for solutions of stochastic differential equations and its applications, J. Math. Kyoto Univ. 13 (1973) 497-512.

[19] J.A., Yan, A comparison theorem for semimartingales and its applications, Séminaire de Probabilités, XX, Lecture Notes in Mathematics, vol. 1204, Springer, Berlin, 1986, pp. 349-351. 